2010-2013, approximately 75 patients were treated for poisoning by venomous creatures. The majority of these were residents of Far North Queensland, but 10 international and 7 interstate tourists per year experienced poisoning of this nature. Mechanism and patterns of injury (e.g., most frequent injuries sustained) in relation to tourist status, age group and gender will be presented in further detail at the conference.

Conclusions This is a descriptive epidemiological study of injuries sustained by residents and tourists of Far North Queensland. These data can be used to inform injury prevention strategies, and to adequately prepare visitors and residents for safe enjoyment of the region.

\section{CHILDREN AND ADOLESCENTS ADMITTED TO A DANISH LEVEL 1 TRAUMA CENTRE 2002-2011}

Danny Stefan Ekström, Rasmus Hviid Larsen, Jens Martin Lauritsen and Christian Faergemann. Accident Analysis Group, Department of Orthopaedics and Traumatology, Odense University Hospital

\subsection{6/injuryprev-2016-042156.947}

Background Prevention of mortality and severe injury following trauma requires unbiased epidemiological surveillance. The epidemiology of children or adolescents admitted to a Danish trauma centre is largely unknown in particular in relation to home- and leisure risk areas. To describe the epidemiology and severity of potential severely injured children and adolescents admitted to the level 1 trauma centre at Odense University Hospital, Denmark.

Methods A descriptive study including all children and adolescents aged 0-17 admitted to the level 1 trauma centre at Odense University Hospital, Denmark 2002-2011. Data was extracted from the multiple trauma register and medical records. The Abbreviated Injury Score (AIS) was calculated in each patient.

Results 950 children and adolescents were included. The median age was 13 (0-17) years. Boys accounted for $60.6 \%$ of the cases. Accidents accounted for $97.2 \%$, violence $1.4 \%$, and self-inflicted injuries $0.4 \%$. More than $3 / 4$ of the injuries occurred either in traffic or at home. The occurrence was greatest in the summer $(34.0 \%)$, during weekends $(48.9 \%)$, and in the hours between 12.00 and 20.00 (59.2\%). 58.5\% of the injuries were due to traffic. Of these $39.7 \%$ were passengers in motor vehicles, $27.5 \%$ drivers/passengers of a scooter/MC, $21.8 \%$ bicyclists and $10.3 \%$ pedestrians. The median ISS and MAIS was 4 (1-75) and 2 (1-6), respectively. Head/face injuries accounted for $36.5 \%$ and extremities for $30.9 \%$ of all injuries. 153 (16.1\%) suffered from severe injuries (ISS > 15). Altogether, $49(5.2 \%)$ died due to their injuries. The mortality among severely injured was $31.4 \%$.

Conclusions Based on a local trauma register it was possible to describe the epidemiology and severity of potential severely injured children and adolescents admitted to a level trauma centre.

\section{URBAN VERSUS RURAL INJURY DIFFERENCES IN OMAN: RESULTS FROM A PROSPECTIVE TRAUMA REGISTRY}

\footnotetext{
${ }^{1}$ Amber Mehmood, ${ }^{1}$ Katharine A Allen, ${ }^{2}$ Ammar Al Kashmiri, ${ }^{3}$ Ali AlBuasidi, ${ }^{4}$ Abdullah AlManiri, ${ }^{1}$ Kent Stevens, ${ }^{1}$ Adnan A Hyder. 'Johns Hopkins School of Public Health, Baltimore, USA; ${ }^{2}$ Khoula Hospital, Muscat, Oman; ${ }^{3}$ Nizwa Hospital, Nizwa, Oman; ${ }^{4}$ The Research Council, Oman
}

Background Oman is a high-income country located in the Arab Gulf Region. The coastal regions of this country consist of highly developed urban centres, with the interior regions being rural and less developed. To-date little data has been generated on the regional differences in injury scale and case mix. Understanding these differences will allow for effective injury-prevention policies and targeted interventions.

Methods A prospective trauma registry was launched in two hospitals in Oman: Khoula Hospital, the national referral centre located in Muscat and Nizwa Hospital, a regional centre serving rural communities. All patients who were admitted with a history of trauma between October 2014 and April 2015 were included. Data was captured on injury details, demographics and treatment received. Further analysis was conducted by hospital of admission.

Results 2,596 cases were included, 62\% were captured at Khoula. Age and sex distribution were similar across both sites. The leading cause of injury at Khoula was falls (39\%) versus transport crashes in Nizwa (38\%). Pedestrians were commonly injured in Muscat (17\%) vs. Nizwa (6\%). Approximately $22 \%$ of cases at Nizwa reached the hospital in less than 30 minutes since time of injury compared to less than $5 \%$ in Muscat. $61 \%$ of patients in Nizwa were transferred from other centres for definitive treatment as compared to Khoula (35\%).

Conclusions Important differences exist between these two hospitals of Oman. A greater proportion of falls in Muscat might reflect the greater number of construction projects. In Nizwa, a rural environment highlights the burden of road injuries. More inter-facility transfers in Nizwa were observed due to initial triage of the victims to the nearest facility. These findings suggest that different injury prevention strategies and trauma care priorities are needed for each location. Additionally, significant differences in hospital transport times highlight the need for improved prehospital care.

\section{THE CHARACTERISTIC AND TREND OF INJURY MORTALITY IN CENTRAL-CHINA, 2008-2012}

'Zhang Qingjun, 'Zhang Lan, ${ }^{1}$ Li Qian, ${ }^{1}$ Pan Jingju, ${ }^{2} Y a n g$ Jiayu. ${ }^{1}$ Hubei Province Centrefor Disease Control and Prevention, China; ${ }^{2}$ School of Public Health, Wuhan University, China

\subsection{6/injuryprev-2016-042156.949}

Background Injury is now an emerging public health problem in China. Hubei Province, located in the central of China, has an area of $185,900 \mathrm{~km}^{2}$ and a population of 57.9 million. Injury is the fourth leading cause of death both in Hubei Province and in China. Given the similar injury mortality situation, this study result will be useful to offer reasonable strategies in China.

Methods The injury mortality rate, distribution of rate by gender and age, potential years of life lost (PYLL) were adopted to analyse injury mortality data from the Death Registry System in Hubei Province during 2008-2012.

Results The proportions of annual injury deaths were $10.59 \%, 10.26 \%, 9.62 \%, 9.92 \%$ and $9.16 \%$ respectively during 2008-2012.The mortality rates were $60.54 / 10^{5}, 60.79 / 10^{5}$, $61.41 / 10^{5}, 59.72 / 10^{5}$ and $59.81 / 10^{5}$ respectively from 2008 to 2012.The standardised mortality rates were $53.21 / 10^{5}, 52.34 /$ $10^{5}, 52.19 / 10^{5}$ and $48.96 / 10^{5}$ respectively. The male mortality rates were $72.63 / 10^{5}, 71.70 / 10^{5}, 68.33 / 10^{5}, 77.59 / 10^{5}$ and $73.82 / 10^{5}$ while the female mortality rate were $47.49 / 10^{5}$, $49.10 / 10^{5}, 44.35 / 10^{5}, 44.81 / 10^{5}$ and $44.86 / 10^{5}$ respectively during 2008-2012. Besides, injury mortality comprised about $9.88 \%$ 